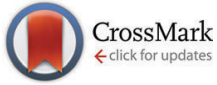

Cite this: J. Mater. Chem. C, 2016, 4, 2828

Received 16th September 2015, Accepted 30th October 2015

DOI: $10.1039 / c 5 t c 02954 f$

www.rsc.org/MaterialsC

\section{Tuning BODIPY molecular rotors into the red: sensitivity to viscosity vs. temperature $\uparrow$}

\author{
Thanh Truc Vu, ${ }^{a}$ Rachel Méallet-Renault, ${ }^{b}$ Gilles Clavier, ${ }^{c}$ Boris A. Trofimov ${ }^{d}$ and \\ Marina K. Kuimova*a
}

Viscosity variations in the microscopic world are of paramount importance for diffusion and reactions. In the last decade a new class of fluorescent probes for measuring viscosity has emerged termed 'molecular rotors', which allows quantitative mapping of viscosity in microscopically heterogeneous environments. Here we attempt to tune the absorption and emission of one such 'molecular rotor' based on the BODIPY fluorescent core into the red region of the spectrum, to allow better compatibility with the 'tissue optical window' and imaging of cells and tissues. We consequently find that our redemitting BODIPY fluorophores are sensitive to environmental temperature rather than to viscosity, thus suggesting a new prototype for a 'molecular thermometer'.

\section{Introduction}

Measuring viscosity on the microscopic scale is of paramount importance for understanding of mechanisms of many processes including diffusion and bimolecular reactions. Molecular rotors $^{1,2}$ have proved their usefulness as small synthetic fluorophores in which fluorescence emission is sensitive to the viscosity of the surrounding environment. This sensitivity, linked to the viscosity dependence of the non-radiative decay of the excited state, allows a precise calibration of fluorescence quantum yield or fluorescence lifetime with viscosity. ${ }^{1}$

For molecular rotors it is now well established that in order to achieve quantitative measurements and, in particular, imaging of viscosity without any bias associated with the variable concentration of the probe, a ratiometric mode of signal detection is required. ${ }^{1}$ For this purpose either the ratio of two fluorescent peaks of the rotor or the fluorescence lifetime measurements (that are intrinsically ratiometric) can be used. Fluorescence lifetime detection of molecular rotors offers advantages due to ease of system calibration, ultrasensitive detection and the possibility to be coupled with imaging (Fluorescence Lifetime Imaging, FLIM), and in recent years it

\footnotetext{
${ }^{a}$ Chemistry Department, Imperial College London, Exhibition Road, SW7 2AZ, UK. E-mail:m.kuimova@imperial.ac.uk

${ }^{b}$ Institut des Sciences Moléculaires d'Orsay, ISMO, UMR 8214, CNRS, Université Paris Sud, 91405 Orsay Cedex, France

${ }^{c}$ PPSM, ENS Cachan, CNRS, UniVerSud, 61 aV President Wilson, F-94230 CACHAN, France

${ }^{d}$ A. E. Favorsky Irkutsk Institute of Chemistry, Siberian Branch of the Russian Academy of Sciences, 1 Favorsky Str., Irkutsk 664033, Russian Federation

$\dagger$ Electronic supplementary information (ESI) available: Synthetic characterisation and additional spectroscopic data for 2-4. See DOI: 10.1039/c5tc02954f
}

has proved extremely successful in detecting microviscosity in many heterogeneous objects, and in particular in individual organelles of live biological cells. ${ }^{1,3-8}$

One of the most widely used lifetime-based molecular rotors is phenyl-BODIPY (Boron DIPYrromethene) $1,{ }^{1,3,4}$ which possesses several convenient properties for a molecular rotor, namely (i) lifetime sensitivity in a wide viscosity range, 10-5000 cP, (ii) good dynamic range of lifetimes corresponding to this viscosity range, and (iii) monoexponential decays of the excited state that allows straightforward data interpretation. Furthermore, any secondary detrimental photophysical or photochemical processes such as aggregation and quenching can be easily detected in $\mathbf{1}$, since they result in biexponential or even more complex excited state decay. Moreover, we have recently demonstrated that the parent phenyl-BODIPY rotor provides temperature-independent measurements of viscosity, ${ }^{9,10}$ i.e. population of the 'bright' $v s$. 'dark' excited state levels is not altered by temperature. This temperature independence is an extremely useful property for measuring viscosity in a 'living system', where temperature gradients can occur along with viscosity gradients. Consequently, phenyl-BODIPY-based molecular rotors such as $\mathbf{1}$ have been successfully used to probe the local rigidity of live biological cells, ${ }^{3,4,11-13}$ lipid bilayers ${ }^{10,14}$ and monolayers, ${ }^{15}$ atmospheric aerosols,${ }^{16}$ polymers ${ }^{17}$ and ionic liquids ${ }^{18}$ in some cases at variable temperature.

However, one of the perceived disadvantages of the rotor $\mathbf{1}$ is its emission in the green region of the spectrum (excitation maximum $495 \mathrm{~nm} /$ emission maximum $520 \mathrm{~nm}),{ }^{1,3,4}$ similar to that of fluorescein and GFP. On the one hand, the absorption and emission of 1 in the green precludes deep tissue imaging in biological settings, due to the poor overlap with the 'tissue optical window'. ${ }^{19,20}$ 
On the other hand, simultaneous imaging of 1 with other useful fluorophores (such as for example GFP/fluorescein) is not possible, due to complete spectral overlap. Here we set out to design red-emitting molecular rotors based on the phenylBODIPY, by extending the conjugation in the BODIPY fluorophore. We tested the spectroscopic properties of the new probes in solutions of varying viscosity, temperature and polarity. We concluded that the extended conjugation in phenyl-BODIPYs confers sensitivity to temperature rather than to viscosity, thus allowing us to suggest a new prototype for a BODIPY-based 'molecular thermometer'.

\section{Methods}

\section{Materials}

All solvents were spectroscopic grade from Sigma-Aldrich and were used as received without further purification. BODIPY 4 was synthesised as previously described. ${ }^{21}$ BODIPYs 2 and 3 were synthesised by adaptation of the literature procedure. $^{21}$ The details of their synthesis and the corresponding ${ }^{1} \mathrm{H}$ and ${ }^{13} \mathrm{C}$ NMR characterisation data are given in the ESI, $\dagger 1 \mathrm{mM}$ solutions of 2 in dichloromethane and of 3, 4 in chloroform were used as stock solutions for all measurements, to achieve a final concentration of BODIPYs of $3 \mu \mathrm{M}$. The viscosity calibration was performed in Baysilone Fluids M Silicone oils 100, 500 and 1000 by varying the temperature between 0 and $100{ }^{\circ} \mathrm{C}$, covering a viscosity range between 30 and $1850 \mathrm{cP}$, according to the manufacturer's specifications. In order to allow a more precise calibration, the viscosities of Silicone oils at precise temperatures used for spectroscopic data collection were measured using an SVM 3000 viscometer (Anton Paar) with 1\% accuracy.

\section{Absorption and fluorescence spectra, quantum yield measurements}

Quartz cuvettes with a $10 \mathrm{~mm}$ path length were used in all measurements. Absorption spectra were measured using an Agilent 8453 UV-Vis spectrophotometer. Fluorescence spectra and quantum yields were recorded using a Fluoromax- 4 spectrofluorometer (Jobin-Yvon; Horiba). Temperature in the cuvette was controlled by a Peltier thermostated cuvette holder (F3004, JobinYvon; Horiba) to $0.5{ }^{\circ} \mathrm{C}$ accuracy, and was additionally verified by inserting a thermocouple (2006T, Digitron) into the cuvette. The quantum yields of $\mathbf{2 - 4}$ were determined by comparative method relative to Rhodamine 101 in ethanol $(+0.01 \% \mathrm{HCl})$ as a standard $\left(\phi_{\mathrm{f}}=1\right)^{22}$ and adjusted for variations in the refractive index of each solvent used. Estimated measurement errors were $1 \%$ (relative quantum yield) and 5\% (absolute quantum yield).

\section{Fluorescence decay measurements}

Fluorescence decays were measured using a DeltaFlex TCSPC device (Horiba) with $560 \mathrm{~nm}$ NanoLED as an excitation source (1.3 ns pulse duration, Horiba). The time window was $100 \mathrm{~ns}$ with 4096 time bins. The decays were accumulated until 10000 counts were reached at the peak. Temperature was controlled by a circulating thermostat (RE104, Lauda Technology Ltd.) to $1{ }^{\circ} \mathrm{C}$, and was additionally verified by inserting a thermocouple (2006T, Digitron) into the cuvette. All decays could be fitted using a monoexponential decay function with $1.0<\chi^{2}<1.2$. Based on the total number of counts recorded, estimated measurement errors in fluorescence lifetime determination were less than $1 \%$.

\section{Results and discussion}

It is now well established that introducing symmetric extended conjugation in the alpha position of BODIPY fluorophores, such as with a phenyl, alkenyl or alkynyl substituent, results in a significant red shift in BODIPY's absorption and emission spectra. ${ }^{21,23,24}$ Therefore we designed phenyl-BODIPYs 2 and 3, analogous to $\mathbf{1}$, which incorporate phenanthrene (2) and naphthalene (3) substituents, in order to shift their absorption/ emission spectra to lower energies that better coincide with the tissue optical window, Fig. 1. The derivative $\mathbf{4}$ was also synthesised as a control fluorophore that is not a molecular rotor, since in 4 the phenyl group rotation thought to be responsible for the molecular rotor function is restricted due to the presence of the methyl substituents on the phenyl group. ${ }^{3}$

We measured absorption and fluorescence spectra of all the new BODIPYs in a non-viscous acetonitrile solution, Fig. 2. As expected, both the absorption and the emission spectra of all dyes shifted significantly to the red region of the spectra compared to the parent rotor $\mathbf{1}$. We also measured absorption

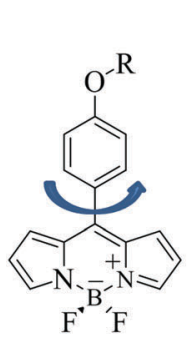

1

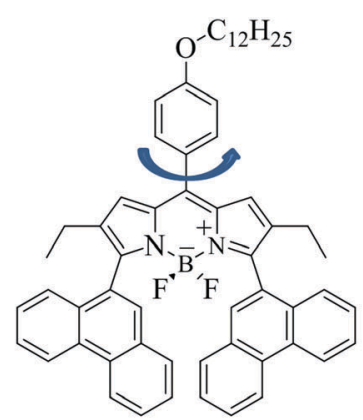

2

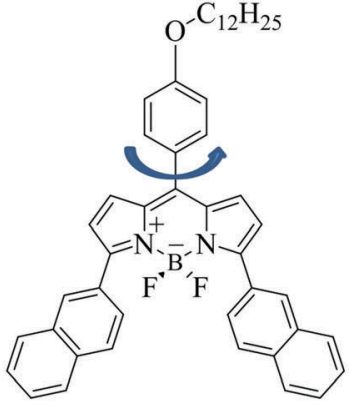

3

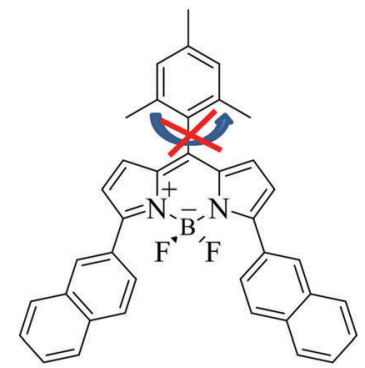

4

Fig. 1 Chemical structure of phenyl-BODIPY derivatives under study. 

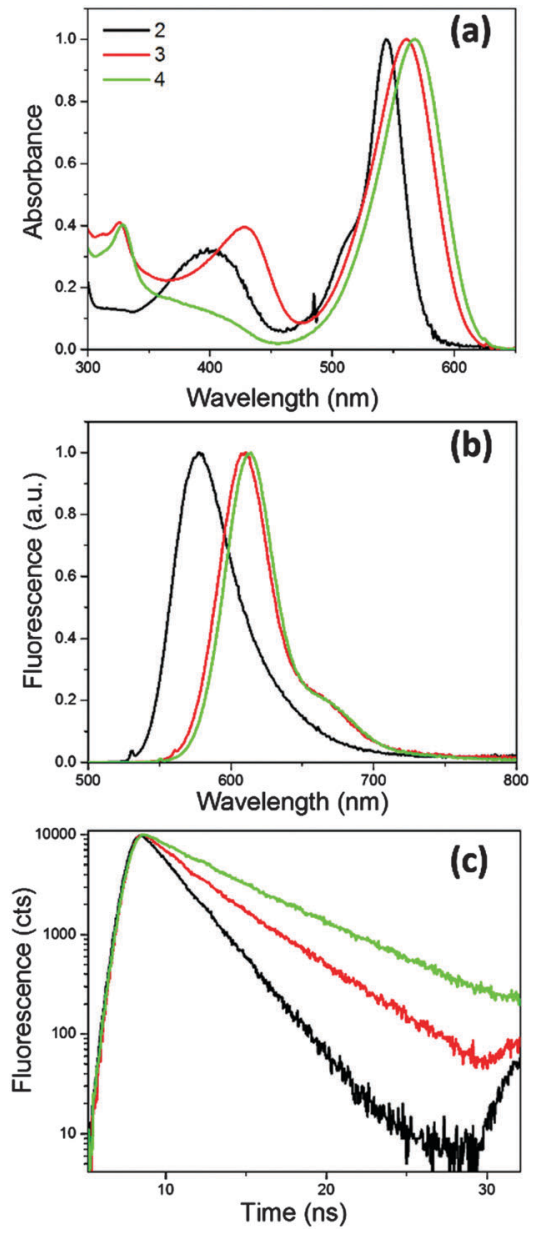

Fig. 2 (a) Absorption, (b) fluorescence spectra and (c) time-resolved fluorescence decay traces recorded for 2-4 in acetonitrile solutions at $25{ }^{\circ} \mathrm{C}$. Excitation wavelength $560 \mathrm{~nm}$.

and emission spectra of 2-4 in a series of solvents of different polarity, and confirmed that the absorption and emission maxima do not display any significant solvatochromism (Fig. S1 and Tables S1 and S2, ESI $\dagger$ ). The fluorescence quantum yields of 2-4 measured in acetonitrile were $0.33,0.53$ and 1.00 respectively. The fluorescence decay traces could be fitted using monoexponential function and yielded fluorescence lifetimes of 2.2, 3.6 and $5.5 \mathrm{~ns}$, for 2-4, respectively. The absorption and emission spectra in all solutions did not display any signs of aggregation; likewise, the monoexponential nature of the excited state decays gave evidence for the absence of aggregation in all solutions.

The quantum yield and the lifetime data are consistent with our hypothesis that $\mathbf{4}$ is not a molecular rotor, and that the incorporation of a mesityl instead of a phenyl substituent on a BODIPY core has completely prevented the rotation of this group and hence blocked the non-radiative decay. Importantly, both 2 and 3 have shorter excited state lifetimes than 4, and quantum yields significantly less than unity, which suggests that the non-radiative deactivation of the excited state in $\mathbf{2}$ and 3 is significant.
Next, we tested the behaviour of 2-4 in mixtures of different viscosity, Fig. 3. Due to the low solubility of these dyes in polar solvents, we chose to perform calibration in a range of silicone oils, commonly used as standards for calibration of rheometers and viscometers. For example, Silicone oil 1000 changes its viscosity between 300 and $1600 \mathrm{cP}$ upon temperature change between 4 and $100{ }^{\circ} \mathrm{C}$, thus providing an excellent dynamic range for the calibration of the new BODIPYs.

As expected for a molecular rotor, 2 shows a very strong dependence of the fluorescence quantum yield and lifetime on viscosity. We note that in this experiment the change in viscosity was brought about by temperature variations. Both photophysical parameters, the quantum yield and the lifetime, display a good dynamic range, between 0.3-0.9 $\left(\Phi_{\mathrm{f}}\right)$ and between 2.5-5.5 ns $\left(\tau_{\mathrm{f}}\right)$, Fig. 3(e and f). Similarly, the quantum yield and lifetime of $\mathbf{3}$ changed significantly upon the temperature change (Fig. 3e and f), although with a slightly narrower dynamic range than for the derivative 2 , indicating that $\mathbf{2}$ is a more sensitive rotor. The fluorescence spectra and the time resolved decay traces recorded for the non-rotor $4 \mathrm{did}$ not show any change upon varying temperature/viscosity of the Silicone oil (Fig. 3).

We calculated the radiative and the non-radiative decay constants for 2 following a temperature change between 3 and $100{ }^{\circ} \mathrm{C}$. Consequently we found, as expected for a molecular rotor, that the radiative decay stays constant upon the temperature change, while the non-radiative decay increases dramatically at high temperatures, Fig. 4. By analogy with $1{ }^{4}$ we assign the increase in the non-radiative decay in $\mathbf{2}$ at higher temperature to the increased effectiveness of rotation of the phenyl ring in the meso-position of BODIPY, which becomes significantly more likely in a low viscosity/high temperature environment.

Having confirmed that the new derivatives 2 and 3 do indeed behave in a similar manner to the molecular rotor $\mathbf{1}$, we set out to extend the calibration range of these new dyes. For this purpose we investigated the photophysics of 2 and 3 in an extended range of silicone oils, e.g. Silicone oil 100 and Silicone oil 500. These are lower molecular weight oils with polarity identical to that of Silicone oil 1000, but with different viscosity ranges attained via temperature change between 0 and $100{ }^{\circ} \mathrm{C}$. The ranges are 30 to $170 \mathrm{cP}$ for Silicone oil 100 and 165 to $850 \mathrm{cP}$ for Silicone oil 500, achieved between 0 and $100{ }^{\circ} \mathrm{C}$. The fluorescence quantum yield and the lifetime data recorded for 2 in these solvents is shown in Fig. 5. Unexpectedly, it appears that the data from all silicone oils overlap perfectly, independent of their viscosity; instead, they appear to depend on temperature alone.

To investigate this further we performed temperaturedependent measurements of fluorescence quantum yield and viscosity in methanol and in toluene, Fig. 5 . The premise here was that the viscosity of both solutions $(\mathrm{ca} .0 .6 \mathrm{cP}$ at room temperature) varies very little with temperature, and thus studying the photophysics in these solvents should allow us to decouple the effect of viscosity from that of temperature. Consequently, we found that the quantum yield values recorded for $\mathbf{2}$ in toluene solutions at varying temperature 

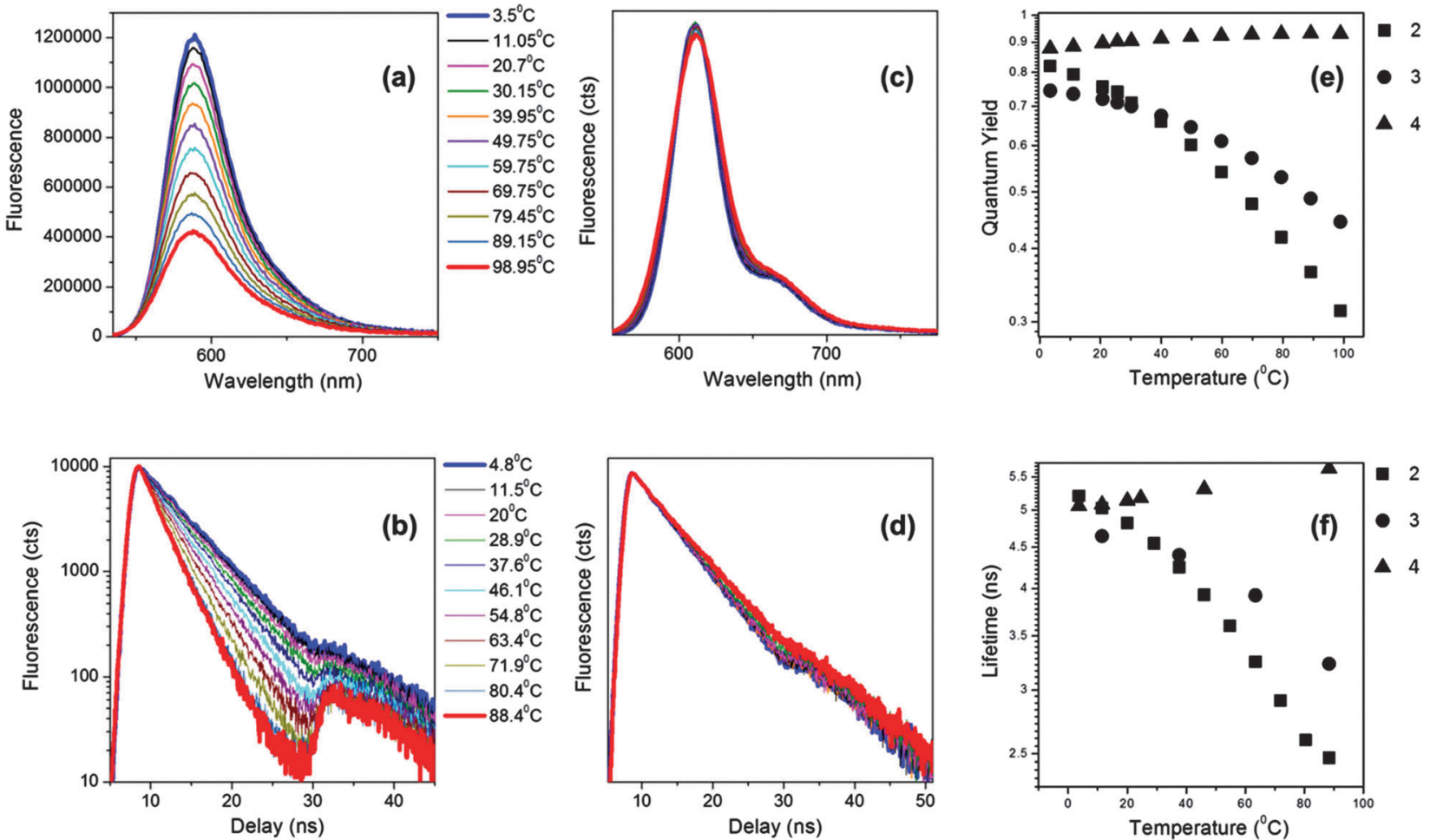

Fig. 3 Fluorescence spectra ( $a$ and $c$ ) and time resolved fluorescence decays (b and d) of compounds 2 ( $a$ and b) and $\mathbf{4}$ (c and d) in Silicone oil 1000 recorded upon a temperature change between 3.5 and $99^{\circ} \mathrm{C}$. Quantum yields (e) and fluorescence lifetimes (f) recorded for compounds $\mathbf{2}, 3$ and 4 in Silicone oil 1000 upon a temperature change between 3.5 and $99{ }^{\circ} \mathrm{C}$. Excitation wavelength $560 \mathrm{~nm}$, detection wavelength $589 \mathrm{~nm}$ (b) and $612 \mathrm{~nm}(\mathrm{~d})$.

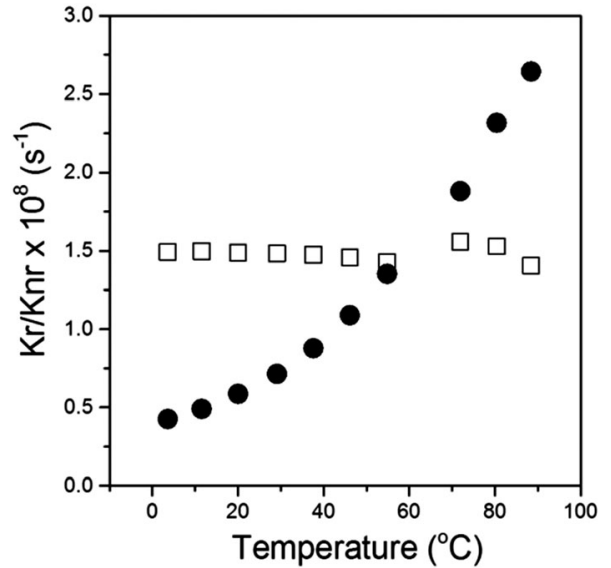

Fig. 4 Radiative $(\square)$ and non-radiative $(\bullet)$ decay constants calculated for 2 in Silicone oil 1000 upon a temperature change between 3.5 and $99^{\circ} \mathrm{C}$.

overlaps perfectly with the data for silicone oils 100, 500 and 1000. While the fluorescence quantum yields and lifetimes recorded for 2 in methanol are somewhat lower than those in toluene, the trend observed upon changing the solution temperature is very similar to that observed in other solvents, i.e. both the $\Phi_{\mathrm{f}}$ and the $\tau_{\mathrm{f}}$ values decrease dramatically at higher temperatures.

Finally, we recorded fluorescence quantum yields and lifetimes for 2 in mixtures of methanol/glycerol (50-100\% methanol) at room temperature, where viscosity changes between 0.6 and $15 \mathrm{cP}$, and found a good overlap between all spectra and time resolved traces recorded, Fig. 6. We were unable to investigate higher glycerol content mixtures (with higher viscosity), due to poor solubility of 2 in this media. Still, based on the data presented above we can conclude that the solution viscosity plays a very minor (if any) role in the photophysics of the red-emitting BODIPYs 2 and 3 . Instead, the non-radiative decay of these new dyes appears to be governed by the environmental temperature.

This observation is in stark contrast to our data for the complete lack of temperature dependence in green-emitting BODIPY rotors constructed as $1 .{ }^{9}$ We have previously demonstrated that, independent of a substituent $\mathrm{R}$ (cholesterol, $\mathrm{C}_{12}$ or $\mathrm{C}_{10}$ chain, doubly charged chain), ${ }^{10,13,14}$ the photophysics of 1 is completely independent of temperature and responds to the viscosity alone. On the other hand, 2 and 3 respond only to temperature and their photophysics does not appear to respond to viscosity. At the same time, in both series of fluorophores the mechanism of non-radiative decay activation appears to be through the rotation of the phenyl ring, since the substitution of the phenyl for the mesytyl substituent in $1,,^{3,25} \mathbf{2}$ or 3 causes the rotor/temperature sensor properties to disappear. Likewise, the temperature/viscosity effects work by affecting the non-radiative (rather than the radiative) decay constant, and an increased temperature causes the $k_{\mathrm{nr}}$ to increase in 2,3 (Fig. 4), while a decreased viscosity causes the $k_{\mathrm{nr}}$ to increase in $\mathbf{1 .}^{3,4}$ 

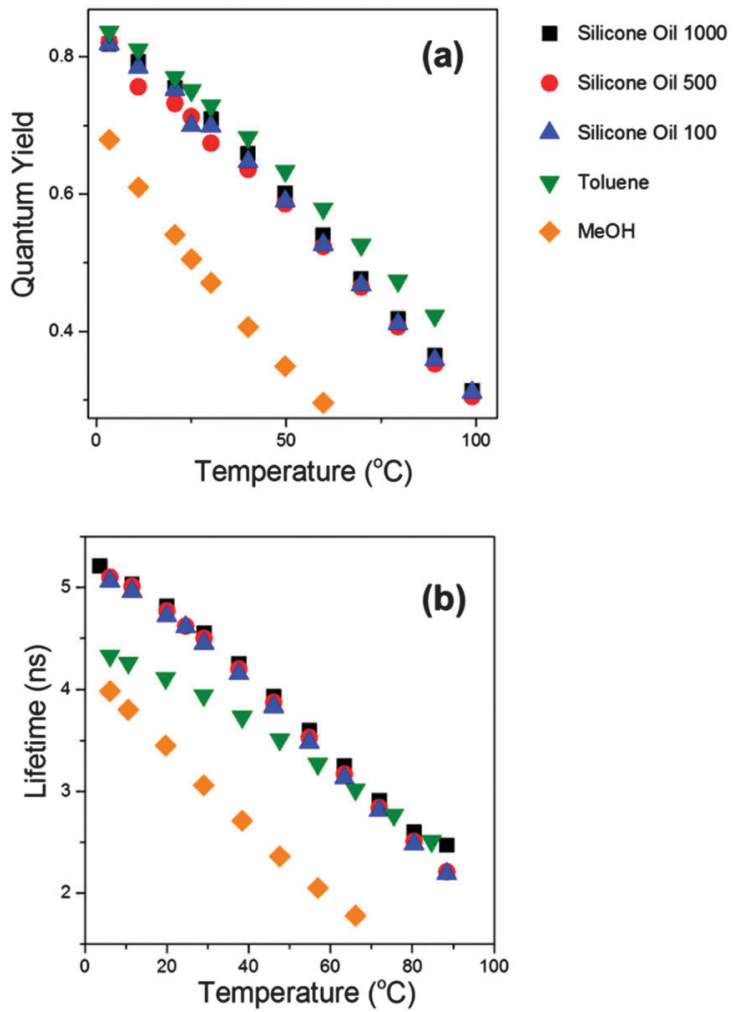

Fig. 5 Fluorescence quantum yields (a) and fluorescence lifetimes (b) recorded for compound $\mathbf{2}$ in various solvents upon a temperature change between 3.5 and $99{ }^{\circ} \mathrm{C}$.

In Fig. 5 we have compared the data recorded for 2 in methanol and toluene, both non-viscous solvents with the same viscosity. Given the difference in the fluorescence quantum yields and lifetimes recorded in these two solvents at the same temperature, and having excluded aggregation as a cause of altered photophysical behaviour, we have hypothesised that solvent polarity might play a role in the photophysics of 2 and 3 . For rotor 1 we have previously established that polarity affects the photophysics of $\mathbf{1}$, but only in solutions of low viscosity. ${ }^{14}$

Thus, we have recorded the fluorescence quantum yields and lifetimes of $\mathbf{2}$ and $\mathbf{3}$ in a series of solvents of varying polarity. In Fig. 7 data is plotted against values of the SdP parameter from Catalan methodology, ${ }^{26}$ where 0 corresponds to non-polar solvents (e.g. cyclohexane) and 1 to polar solvents (e.g. DMSO). The resulting trend is particularly clear for the compound 2, possibly due to the higher dynamic range of fluorescence quantum yield and lifetime covered by this dye. This trend indicates that between SdP 0 and 0.8 polarity does not play a strong role in the photophysics of the red BODIPY dyes 2 and 3. However, it should be noted that for very polar solvents of SdP $>0.8$ both the quantum yield and the lifetime decrease significantly compared to the values in non-polar solvents. This fact reflects the appearance of an additional deactivation channel from the excited state of 2 and 3 , governed by an increase in polarity. It is important to note that even in solvents of increased polarity we observed no spectroscopic signs of aggregation in absorption and emission spectra as
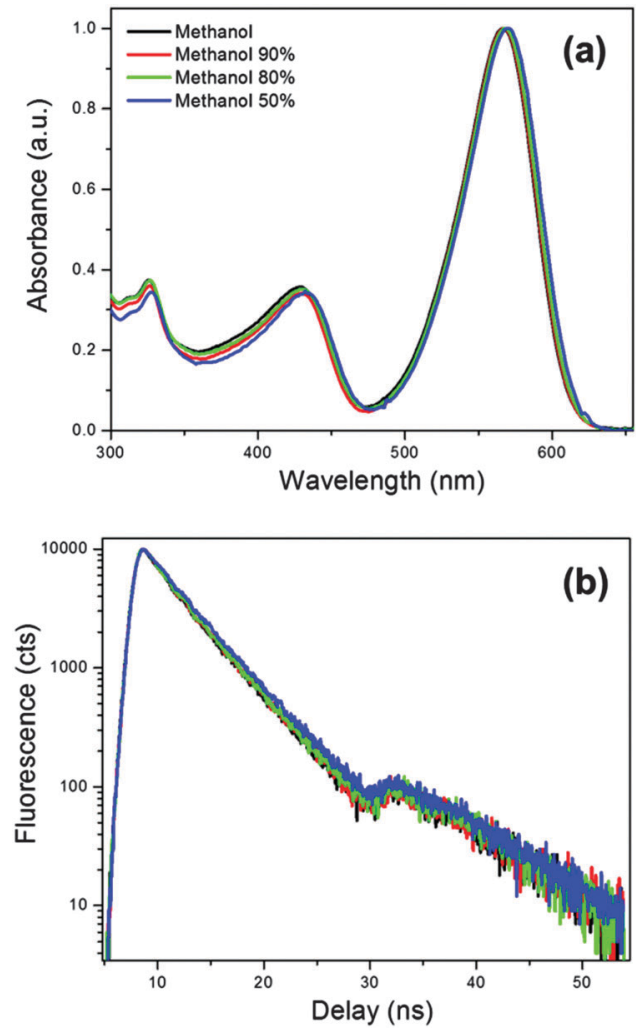

Fig. 6 Absorbance spectra (a) and fluorescence time resolved decay traces (b) recorded for compound $\mathbf{2}$ in methanol/glycerol mixtures at $25{ }^{\circ} \mathrm{C}$. The corresponding quantum yields were 0.57 (methanol), 0.61 (10\% glycerol), 0.63 (20\% glycerol) and 0.71 (50\% glycerol).
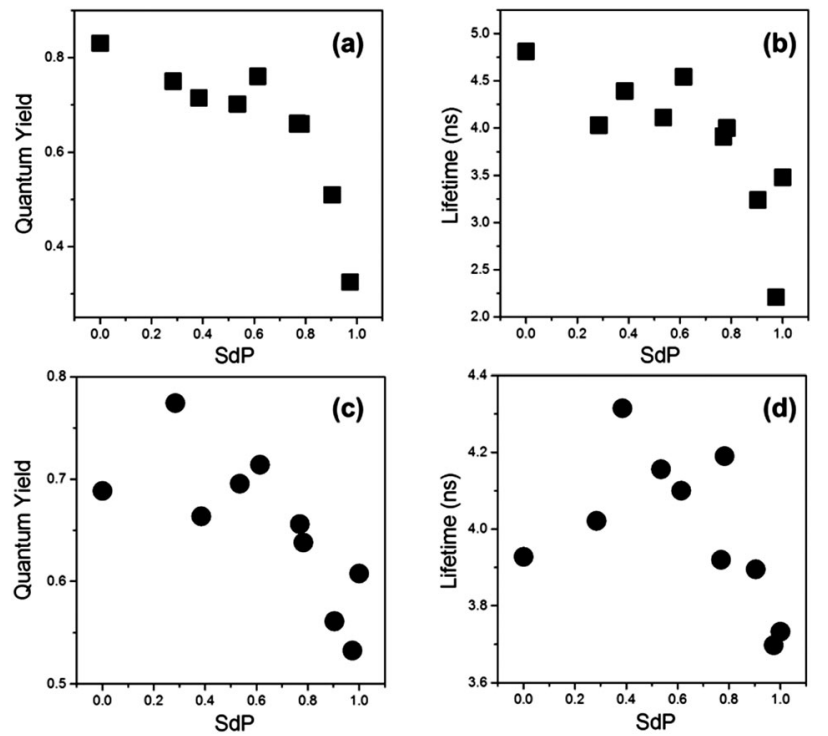

Fig. 7 Fluorescence quantum yields ( $a$ and $c$ ) and fluorescence lifetimes ( $b$ and $d$ ) recorded for compound $\mathbf{2}$ ( $a$ and $b$ ) and $\mathbf{3}$ ( $c$ and $d$ ) in solvents of variable polarity at room temperature. The SdP parameters for all solvents used were taken from ref. 26 and are listed in the ESI, $\uparrow$ Table S4.

well as in the time resolved emission decays that remained monoexponential in all cases. 


\section{Conclusions}

In conclusion, we have synthesised two new red emitting phenyl-BODIPY fluorophores 2 and 3, whose photophysical behaviour strongly responds to the rotation of the phenyl group in the meso-position of the BODIPY. Unlike the green-emitting parent phenyl-BODIPY 1, a so called 'molecular rotor', 1,3,4 whose photophysical parameters are known to respond to the viscosity of its immediate environment, the red-emitting derivatives we have designed here respond to the temperature of their microscopic environment, and not to its micro-viscosity. We believe that the reason for the different temperature sensitivity in BODIPYs $\mathbf{1}$, compared to $\mathbf{2}$ and $\mathbf{3}$, is the relative position of the 'bright' and 'dark' excited states, i.e. a different energy gap between these states. Experimental and theoretical studies to unravel the reason for this interesting behaviour are underway in our laboratory.

Importantly, the temperature appears to be the only parameter strongly affecting the fluorescence quantum yield and the lifetime of BODIPYs 2 and 3, at least in the low-to-medium polarity environment. This is a very useful characteristic of a temperature sensor, and, unlike many other probes currently used, ${ }^{9}$ this means that there is no need to decouple the effect of viscosity from that of temperature when interpreting the data. Thus these new red-emitting BODIPY fluorophores represent a new prototype of a 'molecular thermometer', which should allow the measurement of temperature variations on a microscopic scale.

\section{Acknowledgements}

MKK is thankful to the EPSRC for the Career Acceleration Fellowship (EP/I003983/1). This work was partially supported by the European Commission in the form of a Marie Curie individual Fellowship to TTV under the contract PIEF-GA-2011302441 .

\section{Notes and references}

1 M. K. Kuimova, Phys. Chem. Chem. Phys., 2012, 14, 12671-12686.

2 M. A. Haidekker and E. A. Theodorakis, Org. Biomol. Chem., 2007, 5, 1669-1678.

3 M. K. Kuimova, G. Yahioglu, J. A. Levitt and K. Suhling, J. Am. Chem. Soc., 2008, 130, 6672-6673.

4 J. A. Levitt, M. K. Kuimova, G. Yahioglu, P. H. Chung, K. Suhling and D. Phillips, J. Phys. Chem. C, 2009, 113, 11634-11642.

5 E. Gatzogiannis, Z. Chen, L. Wei, R. Wombacher, Y.-T. Kao, G. Yefremov, V. W. Cornish and W. Min, Chem. Commun., 2012, 48, 8694-8696.
6 X. J. Peng, Z. G. Yang, J. Y. Wang, J. L. Fan, Y. X. He, F. L. Song, B. S. Wang, S. G. Sun, J. L. Qu, J. Qi and M. Yang, J. Am. Chem. Soc., 2011, 133, 6626-6635.

7 N. Jiang, J. Fan, S. Zhang, T. Wu, J. Wang, P. Gao, J. Qu, F. Zhou and X. Peng, Sens. Actuators, B, 2014, 190, 685-693.

8 A. Vyšniauskas, M. Balaz, H. L. Anderson and M. K. Kuimova, Phys. Chem. Chem. Phys., 2015, 7548-7554.

9 A. Vyšniauskas, M. Qurashi, N. Gallop, M. Balaz, H. L. Anderson and M. K. Kuimova, Chem. Sci., 2015, 6, 5773-5778.

10 Y. Wu, M. Štefl, A. Olzyńska, M. Hof, G. Yahioglu, P. Yip, D. R. Casey, O. Ces, J. Humpolíčková and M. K. Kuimova, Phys. Chem. Chem. Phys., 2013, 15, 14986-14993.

11 L. Wang, Y. Xiao, W. Tian and L. Deng, J. Am. Chem. Soc., 2013, 135, 2903-2906.

12 Z. Yang, Y. He, J.-H. Lee, N. Park, M. Suh, W.-S. Chae, J. Cao, X. Peng, H. Jung, C. Kang and J. S. Kim, J. Am. Chem. Soc., 2013, 135, 9181-9185.

13 I. López-Duarte, T. T. Vu, M. A. Izquierdo, J. A. Bull and M. K. Kuimova, Chem. Commun., 2014, 50, 5282-5284.

14 M. R. Dent, I. López Duarte, C. J. Dickson, N. D. Geoghegan, J. M. Cooper, I. R. Gould, R. Krams, J. A. Bull, N. J. Brooks and M. K. Kuimova, Phys. Chem. Chem. Phys., 2015, 17, 18393-18402.

15 N. A. Hosny, G. Mohamedi, P. Rademeyer, J. Owen, Y. Wu, M.-X. Tang, R. J. Eckersley, E. Stride and M. K. Kuimova, Proc. Natl. Acad. Sci. U. S. A., 2013, 110, 9225-9230.

16 J. M. Nölle, C. Jüngst, A. Zumbusch and D. Wöll, Polym. Chem., 2014, 5, 2700-2703.

17 J. D. Kimbal, S. Raut, L. P. Jameson, N. W. Smith, Z. Gryczynski and S. V. Dzyuba, RSC Adv., 2015, 5, 19508-19511.

18 N. A. Hosny, C. Fitzgerald, C. Tong, M. Kalberer, M. K. Kuimova and F. D. Pope, Faraday Discuss., 2013, 165, 343-356.

19 J. Eichler, J. Knof and H. Lenz, Radiat. Environ. Biophys., 1977, 14, 239-242.

20 B. C. Wilson, W. P. Jeeves, D. M. Lowe and G. Adam, Prog. Clin. Biol. Res., 1984, 170, 115-132.

21 E. Y. Schmidt, N. V. Zorina, M. Y. Dvorko, N. I. Protsuk, K. V. Belyaeva, G. Clavier, R. Méallet-Renault, T. T. Vu, A. I. Mikhaleva and B. A. Trofimov, Chem. - Eur. J., 2011, 17, 3069-3073.

22 T. Karstens and K. Kobs, J. Phys. Chem., 1980, 84, 1871-1872.

23 T. Rohand, W. Qin, N. Boens and W. Dehaen, Eur. J. Org. Chem., 2006, 4658-4663.

24 W. Qin, T. Rohand, W. Dehaen, J. N. Clifford, K. Driesen, D. Belijonne, B. Van Averbeke, M. Van der Auweraer and N. Boens, J. Phys. Chem. A, 2007, 111, 8588-8597.

25 K. Yamada, T. Toyota, K. Takakura, M. Ishimaru and T. Sugawara, New J. Chem., 2001, 25, 667-669.

26 J. Catalan, J. Phys. Chem. B, 2009, 113, 5951-5960. 